\title{
ARTICLE The RAGE signaling pathway is involved in intestinal inflammation and represents a promising therapeutic target for Inflammatory Bowel Diseases
}

\author{
M. Body-Malapel ${ }^{1}$, M. Djouina ${ }^{1}$, C. Waxin ${ }^{1}$, A. Langlois ${ }^{1}$, C. Gower-Rousseau ${ }^{1}$, P. Zerbib ${ }^{1,2}$, A.-M. Schmidt ${ }^{3}$, P. Desreumaux $^{1}$, \\ E. Boulanger ${ }^{1}$ and C. Vignal ${ }^{1}$
}

\begin{abstract}
Inflammatory Bowel Diseases (IBD) are chronic inflammatory conditions of the intestinal tract. IBD are believed to result from an inappropriate immune response against the intestinal flora in genetically predisposed patients. The precise etiology of these diseases is not fully understood, therefore treatments rely on the dampening of symptoms, essentially inflammation, rather than on the cure of the disease. Despite the availability of biologics, such as anti-TNF antibodies, some patients remain in therapeutic failure and new treatments are thus needed. The multiligand receptor for advanced glycation end-products (RAGE) is a pattern recognition receptor implicated in inflammatory reactions and immune system activation. Here, we investigated the role of RAGE in intestinal inflammation and its potential as a therapeutic target in IBD. We showed that RAGE was upregulated in inflamed tissues from IBD patients compared to controls. Rage $e^{-1-}$ mice were less susceptible to intestinal and colonic inflammation development than WT mice. WT mice treated with the RAGE-specific inhibitor FPS-ZM1 experienced less severe enteritis and colitis. We demonstrated that RAGE could induce intestinal inflammation by promoting oxidative stress and endothelial activation which were diminished by FPSZM1 treatment. Our results revealed the RAGE signaling pathway as a promising therapeutic target for IBD patients.
\end{abstract}

Mucosal Immunology (2019) 12:468-478; https://doi.org/10.1038/s41385-018-0119-z

\section{INTRODUCTION}

Inflammatory Bowel Diseases (IBD) which include Crohn's disease (CD) and ulcerative colitis (UC) are immune-mediated conditions that involve a complex interplay of genetic, immunological, and environmental factors. ${ }^{1,2}$ The gut is continuously facing harmful and beneficial microbes, food antigens and xenobiotics. It is therefore equipped with immune and non-immune cells and soluble mediators to fine tune the balance between pro- and antiinflammatory responses to maintain homeostasis. In IBD, an improper and dysregulated immune response develops against the gut microbiota in genetically predisposed patients. ${ }^{2}$ The precise etiology of these diseases still remains unclear and, in consequence, there is no definitive treatment. A better understanding of their pathophysiology remains necessary to properly treat them. ${ }^{3}$ Traditionally, management of CD and UC has focused on broad spectrum anti-inflammatory and immunosuppressive treatments. In the last 2 decades, a shift toward a pathway-based approach occurred, targeting immunomodulatory molecules with known physiopathological implications. ${ }^{4,5}$ Despite the advent of anti-TNFa, which has constituted a major breakthrough for patients, many patients remain refractory to treatment or lose response over time. ${ }^{6}$ New therapeutics in IBD should not only lead to clinical improvement but more importantly to deep remission which will prevent long-term sequelae. The receptor for advanced glycation end-products (RAGE) is a cell surface multiligand receptor of the immunoglobulin superfamily. RAGE is expressed in all tissues either constitutively or upon inflammation, and is widely localized in many cell types (hepatocytes, smooth muscle cells, neurons, peritoneal mesothelial cells, immune cells, or epithelial cells...). ${ }^{7-10}$ RAGE is considered as a pattern recognition receptor, responding to danger signals and recognizing a threedimensional structure rather than a specific amino acid sequence. ${ }^{11}$ RAGE possesses ligand binding affinity for a wide range of molecules including advanced glycation end products $(\mathrm{AGE}){ }^{12} \beta$-amyloid peptide, ${ }^{13}$ high mobility group box-1 $(\mathrm{HMGB} 1)^{14}$, or S100/calgranulins. ${ }^{15}$ RAGE and its ligands are upregulated in various inflammatory diseases and in cancer. ${ }^{16-19}$ Pro-inflammatory pathways such as nuclear factor kappa B (NF$\mathrm{KB})$, mitogen-activated protein kinase (MAPK) or janus activated kinase/signal transducers and activators of transcription (JAK/ STAT) are activated following engagement of RAGE. ${ }^{20}$ Several studies showing an upregulation of RAGE in chronic inflammatory diseases suggested its involvement maybe not only in perpetuating inflammation and immune response but also in the pathological process. ${ }^{21,22}$ In CD patients, an upregulation of $\mathrm{RAGE}^{23,24}$ and some of its ligands (S100A12, HMGB1) ${ }^{25,26}$ has been observed. In mice, administration of HMGB1 aggravates small intestine injury ${ }^{27}$ and conversely decreasing of HMGB1 by ethyl pyruvate ameliorates colitis. ${ }^{28}$ The evidences of RAGE blockage leading to dampening of intestinal inflammation are only indirect

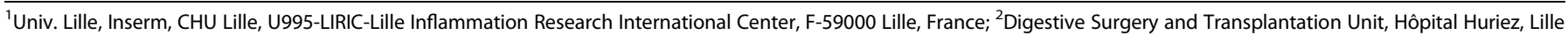

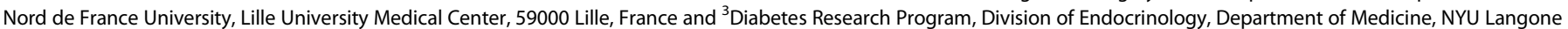
Medical Center, New York, NY 10016, USA

Correspondence: C Vignal (cecile.vignal2@univ-lille.fr)

Received: 21 February 2018 Revised: 25 October 2018 Accepted: 30 October 2018

Published online: 12 December 2018 
a

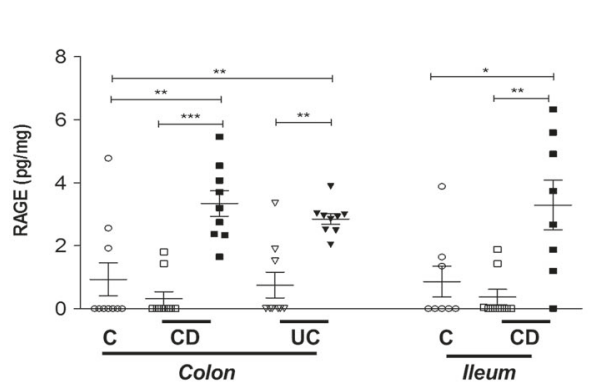

C

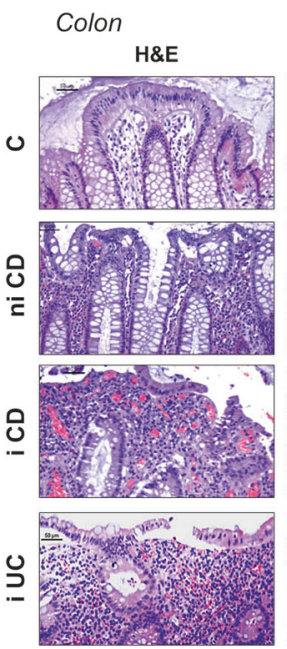

b Ileum

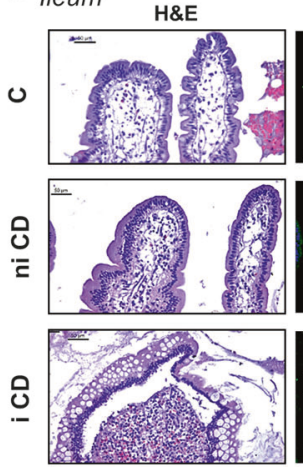

RAGE
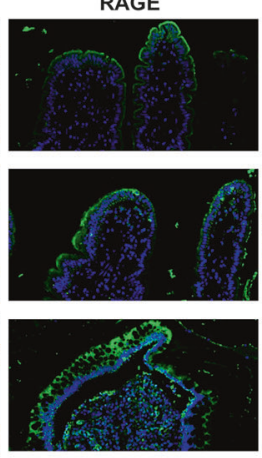

KERATINE 20
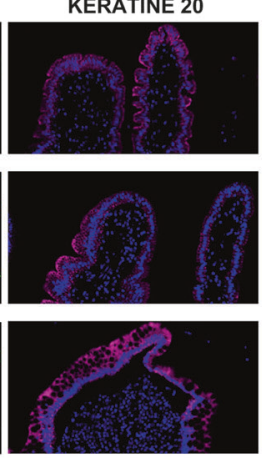

MERGE

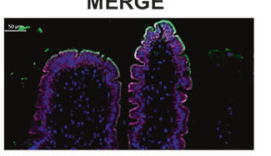

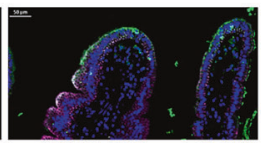
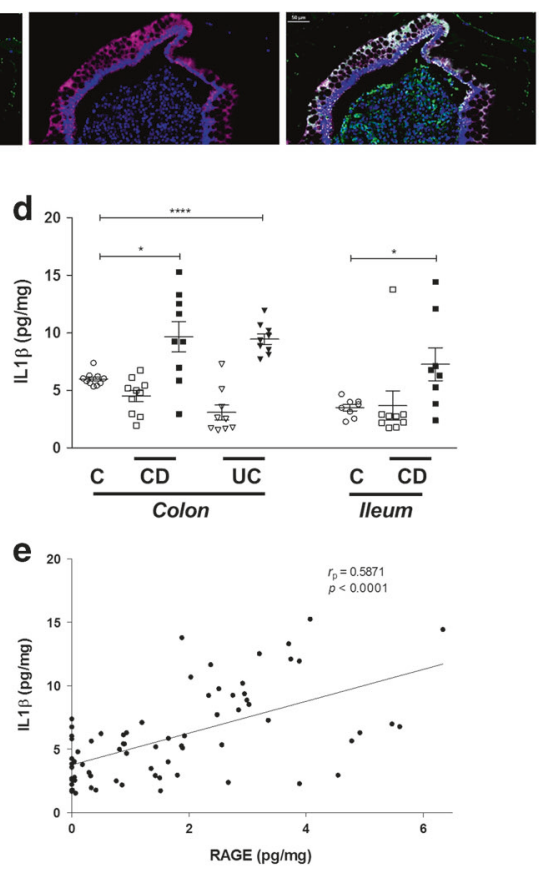

Fig. 1 RAGE is weakly expressed in the colon and ileum of controls and in non-inflamed segments of Crohn's Disease and Ulcerative Colitis patients, but is upregulated under inflammatory conditions. a ELISA quantification of RAGE in the colon and ileum of controls ( $O$ C, $n=8-10)$, in non-inflamed segments of Crohn's Disease ( $\square \mathrm{CD}, n=9-10)$ and Ulcerative Colitis ( $\nabla$ UC, $n=9)$ patients and in inflamed segments of $C D$ ( $\mathbf{~}$, $n=8-9)$ and UC $(\nabla, n=9)$ patients. b Representative hematoxylin/eosin, RAGE and Keratin 20 staining of Ileum of controls (C) and noninflamed (ni CD) and inflamed ( $\mathrm{CD}$ ) segments of CD patients. Scale bar, $50 \mu \mathrm{m}$. c Representative RAGE and Keratin 20 immunostaining of colon of controls (C) and non-inflamed (ni CD) and inflamed (i CD and i UC) segments of CD and UC patients. Scale bar, $50 \mu \mathrm{m}$. d ELISA quantification of IL1 $\beta$ in the colon and ileum of controls (O C, $n=8-10)$, in non-inflamed segments of Crohn's Disease ( $\square$ CD, $n=9-10)$ and Ulcerative Colitis ( $\nabla$ UC, $n=9)$ patients and in inflamed segments of CD $(\mathbf{\square}, n=8-9)$ and UC $(\boldsymbol{\nabla}, n=9)$ patients. e Pearson correlation between RAGE and IL1 $\beta$ quantification in pooled samples of ileum and colon from controls and CD and UC patients. ${ }^{*} p<0.05,{ }^{* *} p<0.01,{ }^{* * *} p$ $<0.005,{ }^{* * * *} p<0.001$ determined by Mann-Whitney test

by blocking RAGE ligands rather than the RAGE itself, ligands that are not RAGE specific. Here, we investigated the implication of RAGE in small intestine and colon inflammation using human gut biopsies and $\mathrm{Rage}^{-1-}$ mice, and the therapeutic potential of the high-affinity RAGE-specific inhibitor FPS-ZM1. ${ }^{29}$

\section{RESULTS}

Rage is expressed in the colon and small intestine of human and mouse and is upregulated under inflammatory conditions The expression of RAGE was assessed in the different segments of the human and mouse digestive tract under steady state and inflammatory conditions.

In human, ELISA quantification revealed that RAGE was barely expressed whether at the colonic or the small intestinal levels in control (C) samples (Fig. 1a; open circles). In non-inflamed areas from CD or UC patients, RAGE was also weakly expressed (Fig. 1a; open squares and triangles). However, an increased expression of RAGE was observed in inflamed areas from the ileum and colon in CD and UC (Fig. 1a; black symbols). These results were confirmed by RAGE immunostaining (Fig. 1b, c and Supplementary Figure S1a and b). In control or in non-inflamed (ni) colonic and ileal samples from CD patients, RAGE was weakly detected and its localization was confined to the epithelial cells, highlighted by Keratin 20 and hematoxylin eosin staining. RAGE staining was increased in inflamed areas of the ileum and colon of CD ( $i C D$ ) and UC ( $i$ UC) patients both in epithelial cells and in immune cells recruited in the lamina propria. Finally, biopsies inflammatory status was evaluated by IL1 $\beta$ and IL8 ELISA quantification and a positive correlation was showed between inflammation and RAGE expression (Fig. 1d, e and Supplementary Figure S1c and d).

In mice, under steady state conditions, RAGE was detected at the mRNA and protein levels all along the digestive tract (Fig. 2a, b; white histograms). RAGE mRNA and protein levels were increased during inflammation; in the colon after TNBS-induced colitis and in the ileum and jejunum after indomethacin-induced enteritis (Fig. 2a, b; black histograms). At the histological level, RAGE immunostaining was weak both in the colon and the small intestine under non-inflamed conditions (Fig. 2c). After indomethacin-induced enteritis, RAGE expression was increased in the jejunum and in the ileum, with a staining in villi, crypts and in the lamina propria. RAGE expression was also increased in the colon following TNBS-induced colitis, both within necrotic area of the epithelium and in the lamina propria. The inflammatory status 


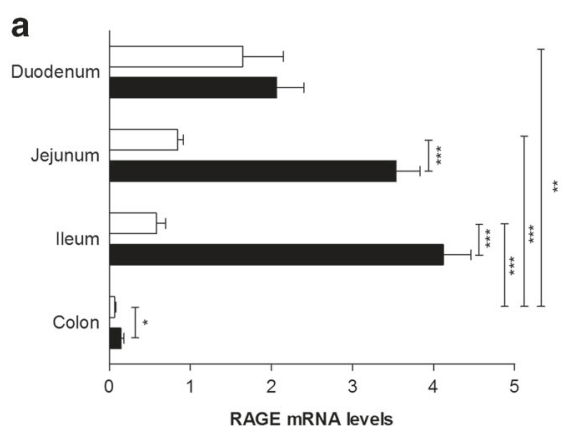

C

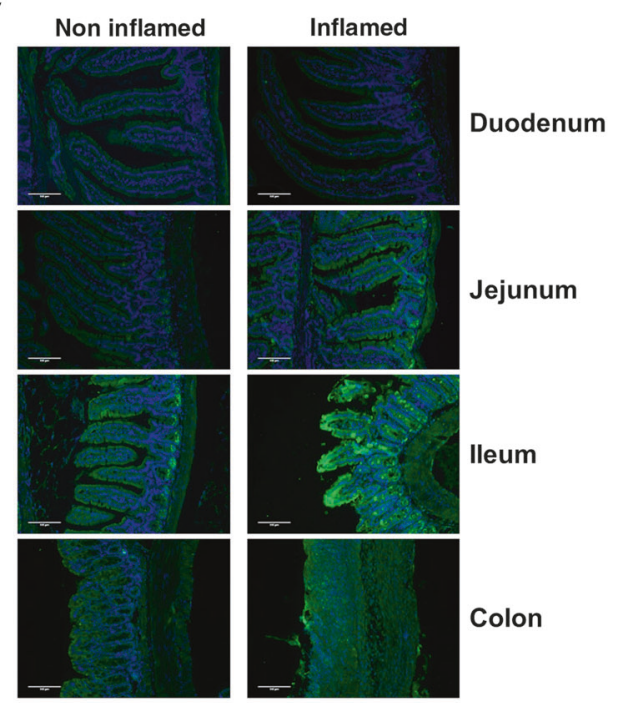

b

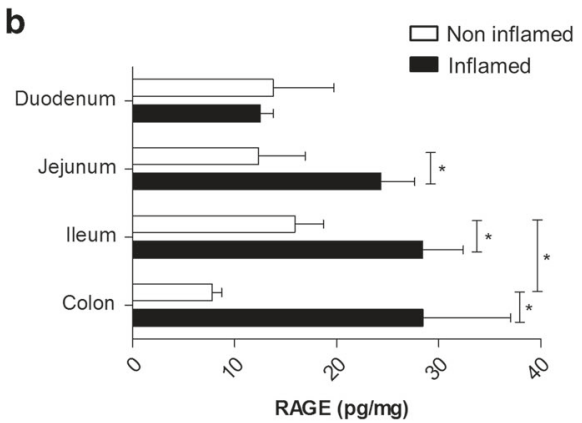

d
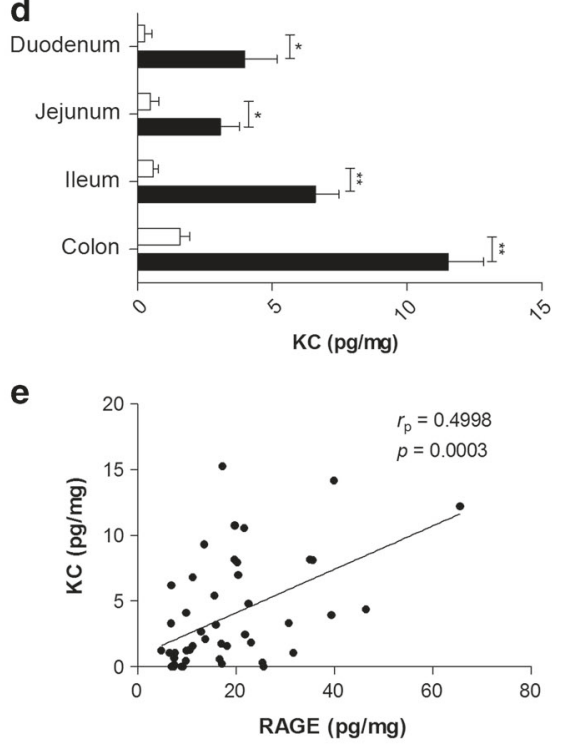

Fig. 2 RAGE is detected from the duodenum to the colon of healthy mice and is upregulated following experimentally induced inflammation. a RAGE mRNA level expression in the duodenum, jejunum, ileum and colon of mice under steady state conditions (white histograms, $n=5$ for small intestinal tissues, $n=10$ for colon) and following experimentally induced inflammation (black histograms, $n=10$ for all segments). $\mathbf{b}$ ELISA quantification of RAGE in the duodenum, jejunum, ileum and colon of mice under steady state conditions (white histograms) and following experimentally induced inflammation (black histograms). c Representative RAGE immunostaining of the duodenum, jejunum, ileum and colon of mice under non-inflamed and inflamed conditions. Scale bar, $100 \mu \mathrm{m}$. d ELISA quantification of KC in the duodenum, jejunum, ileum and colon of mice under steady state conditions (white histograms) and following experimentally induced inflammation (black histograms). e Pearson correlation between RAGE and KC quantification in pooled samples of duodenum, jejunum, ileum and colon from mice under non-inflamed and inflamed conditions. ${ }^{*} p<0.05,{ }^{* *} p<0.01,{ }^{* * *} p<0.005$, determined by Mann-Whitney test

of the mice samples was assessed by ELISA quantification of IL1 $\beta$ and KC and this was correlated with RAGE expression (Fig. 2d, e and Supplementary Figure S2a and b).

Rage $^{-1-}$ mice are protected from indomethacin-induced enteritis and DSS- and TNBS-induced colitis

As RAGE was upregulated in the digestive tract during inflammatory conditions, we wondered if RAGE could have a role in intestinal inflammation development. For this purpose, several models of small intestinal and colonic inflammation were applied to wild-type (WT) and Rage ${ }^{-/-}$mice.

Small bowel inflammation was induced by oral gavage with indomethacin and inflammation was monitored $24 \mathrm{~h}$ later. To allow a better visualization of lesions, Evans blue dye was intravenously injected to mice $30 \mathrm{~min}$ before necropsy. Rage ${ }^{-1-}$ mice developed less severe enteritis in the ileum and jejunum compared to WT mice, duodenum being less affected by indomethacin treatment (Fig. 3a). The number and area of lesions were lowered in the ileum and jejunum of Rage $^{-1-}$ mice compared to WT mice (Fig. 3b, c). Indomethacin administration induced extensive intestinal ulcers and a massive inflammatory infiltrate in the jejunum and ileum of WT mice (Fig. 3d). The lesions were less severe in Rage ${ }^{-1-}$ mice, with a shortening of villus shape, crypt loss and a moderate inflammatory infiltrate. A trend toward a diminution of myeloperoxidase (MPO) activity, a marker of inflammation, and a significant decrease of malondialdehyde (MDA), a marker of lipid peroxidation, were observed in the ileum and jejunum of $\mathrm{Rage}^{-/-}$mice compared to WT mice (Fig. 3e, f). Moreover, IL1 $\beta$ and KC protein levels were decreased in the ileum and jejunum of $\mathrm{Rage}^{-1-}$ mice compared to WT mice (Fig. $3 \mathrm{~g}, \mathrm{~h}$ ).

Colitis was induced in WT and Rage ${ }^{-/-}$mice either by oral DSS or intrarectal TNBS treatment. In both models, Rage ${ }^{-1-}$ mice developed less severe colitis. In the DSS-induced model, weight loss was decreased in Rage ${ }^{-/-}$mice compared to WT mice (Fig. 4a). At necropsy, colon weight/size ratio (Fig. 4b), colonic MPO activity (Fig. 4c) and iNOS and IL1 $\beta$ mRNA levels (Fig. 4d, e) were lowered in Rage $^{-1-}$ mice. In the TNBS model, macroscopic Wallace and histologic ameho scores were lower in the colon of $\mathrm{Rage}^{-/-}$mice, showing ulcerations with deep extension into the muscularis mucosae in WT mice and less extensive ulcerations in $\mathrm{Rage}^{-/-}$mice (Fig. 4f-h). Within lesions, mRNA expression of iNOS and IL1 $\beta$ were minored in Rage R $^{-/-}$ mice (Fig. 4 d, e). Taken together, our results show that Rage ${ }^{-1-}$ mice are less susceptible to gut inflammation, suggesting a role for RAGE in the regulation of intestinal inflammation. 


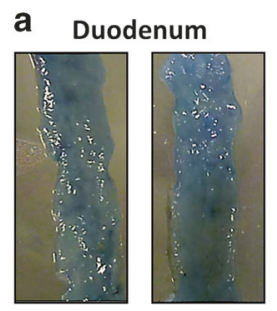

b

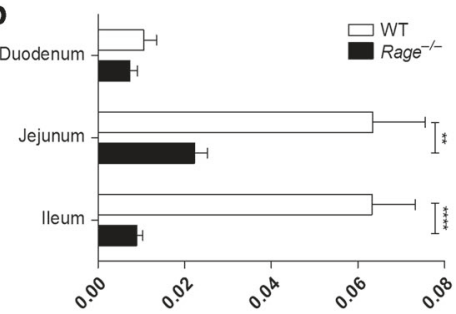

Jejunum

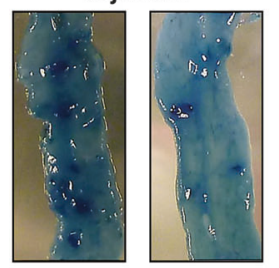

Ileum
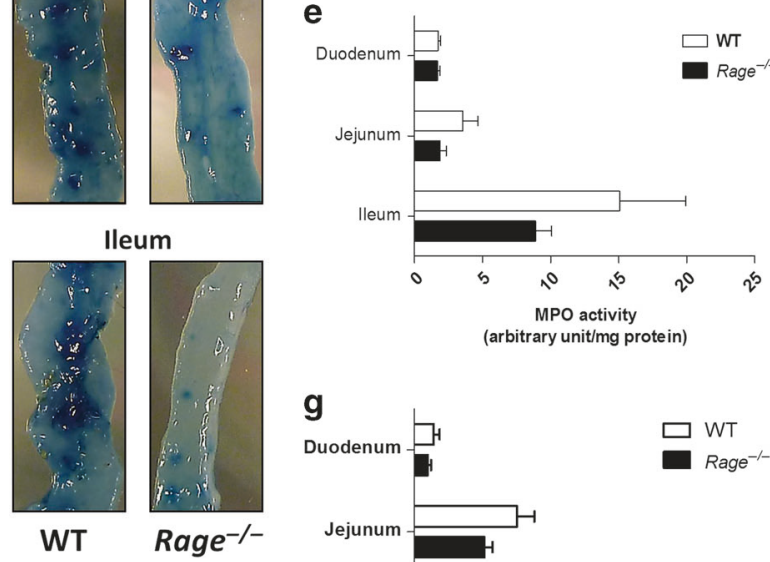

g

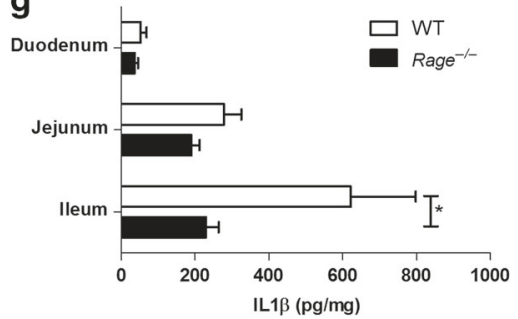

C
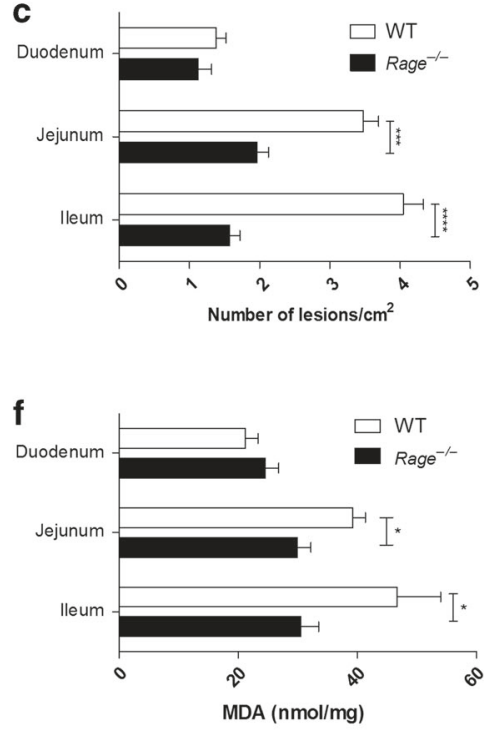

h

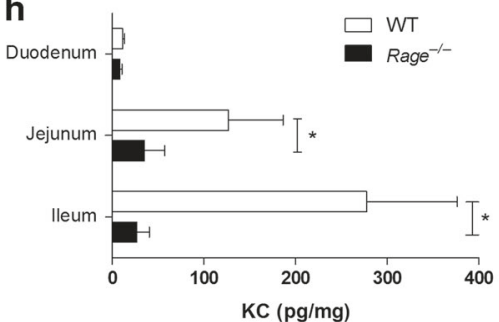

d
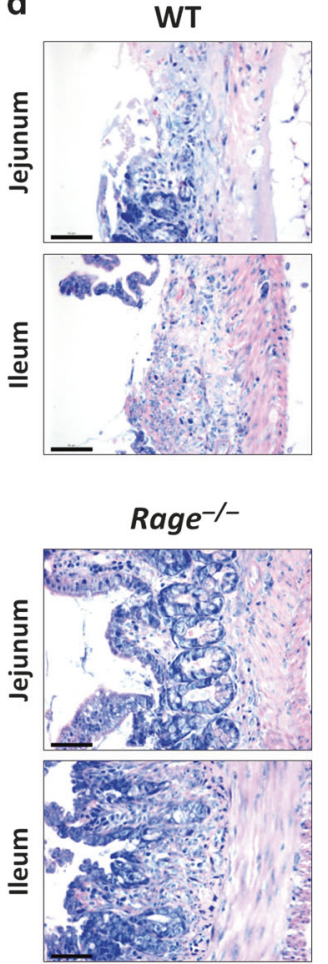

Fig. 3 Rage $^{-/-}$mice are protected from indomethacin-induced enteritis. a Representative pictures of small intestinal lesions. b-g Area (b); and number (c) of lesions; representative pictures of MGG staining of intestinal lesions (scale bar, $50 \mu \mathrm{m})$ (d); quantification of MPO activity (e); and MDA (f); ELISA quantification of IL1 $\beta(\mathbf{g})$; and KC (h) in the ileum, jejunum and duodenum of WT $(n=10)$ and $R^{2}$ following indomethacin treatment. Data are from 5 independent experiments. ${ }^{*} p<0.05,{ }^{* *} p<0.01,{ }^{* *} p<0.005,{ }^{* * * *} p<0.001$ determined by Mann-Whitney test

Given that RAGE has a ubiquitous expression both on immune cells and in the gut epithelium, we wanted to define whether Rage deficiency in hematopoietic versus nonhematopoietic cells was responsible for mice protection during DSS-induced colitis. For this purpose, we set up bone marrow (BM) chimeras, transferring BM either from Rage-deficient donors into wild-type hosts $\left(\right.$ Rage $^{-1-} \rightarrow$ WT) or from WT donors into Rage-deficient hosts (WT $\rightarrow$ Rage $^{-1-}$ ). Both types of chimeras showed lowered histological score compared to WT mice reconstituted with WT BM (WT $\rightarrow$ WT) (Fig. 4i). WT $\rightarrow$ WT mice showed extensive epithelial damages with transmural leukocytes infiltration, in Rage $^{-l-} \rightarrow$ Rage $^{-1-}$ mice and chimeras, mucosal architecture is preserved, inflammatory cells infiltrate persist but less extensively, most often confined to the submucosa (Fig. 4j). Colonic iNos and $I L 1 b$ expression were attenuated in Rage $^{--} \rightarrow$ Rage $^{-/-}$mice and chimeras compared to WT $\rightarrow$ WT mice (Fig. 4k, I). These results revealed that RAGE exerted deleterious functions in both epithelial and hematopoietic cells. However, mice with Rage deficiency in the hematopoietic compartment exhibiting an intermediate phenotype in term of body weight variation (Fig. $4 \mathrm{~m}$ ), it may suggest that RAGE activation in immune cells is particularly important for the development of colitis.

Treatment with the RAGE-specific inhibitor FPS-ZM1 protects mice from indomethacin-induced enteritis and DSS-induced colitis To further explore the role of RAGE and its potential as a therapeutic target in IBD, we tested the effects of the RAGE-specific inhibitor FPS-ZM1 on intestinal inflammation development during indomethacin-induced enteritis and DSSinduced colitis. $^{29}$

The effect of FPS-ZM1 was first assessed on mice under basal condition. Globally, FPS-ZM1 did not induce variations in all tested parameters (Supplementary Figure S3a-k). To evaluate the therapeutic effect of FPS-ZM1 on small bowel inflammation, the RAGE inhibitor FPS-ZM1 was intraperitoneally administrated, at the dosages of 1 and $5 \mathrm{mg} / \mathrm{kg} /$ day, once daily for 9 days before indomethacin gavage, and mice were sacrificed $24 \mathrm{~h}$ after indomethacin administration (Fig. 5a). FPS-ZM1 administration protected mice from indomethacin-induced enteritis with no difference between the 2 dosages of FPS-ZM1 (Fig. 5b). The number and area of lesions were lower in the ileum and jejunum of mice treated with FPS-ZM1 compared to untreated mice (Fig. 5c, d). Consistently, MPO activity, MDA and IL1 $\beta$ and KC levels were decreased in mice treated with FPS-ZM1 compared to nontreated mice (Fig. 5e-h).

In the model of DSS-induced colitis, FPS-ZM1 was intraperitoneally administered to mice at a dosage of 1 and $5 \mathrm{mg} / \mathrm{kg} /$ day during the course of DSS treatment (Fig. 6a). Both FPS-ZM1 dosages protected mice from colonic inflammation. Compared to DSS-treated mice, mice receiving DSS and the RAGE inhibitor did not lose weight during the course of DSS (Fig. $6 \mathrm{~b}$ and Supplementary Figure S4a). The clinical score, gathering stool consistency and occult blood, was less severe in RAGE inhibitortreated mice compared to control DSS mice (Fig. $6 \mathrm{c}$ ). The colon weight/size ratio was lower in mice treated with FPS-ZM1 compared to control DSS mice (Fig. 6d). Consistently, histological score (Fig. 6e and Supplementary Figure S4b), colonic MPO 
a

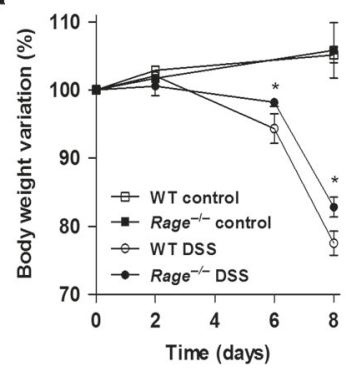

e

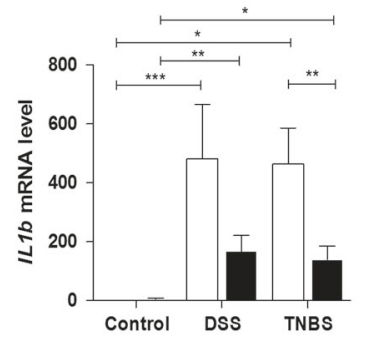

b

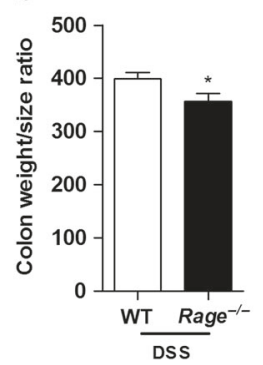

f

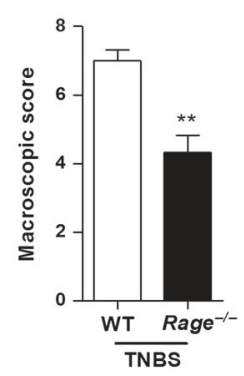

c

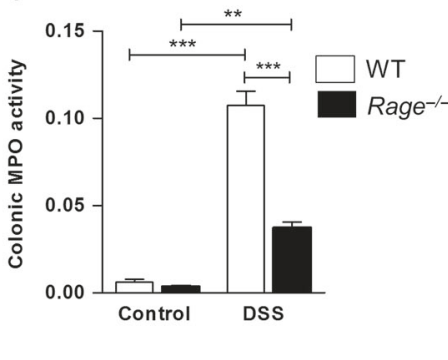

d

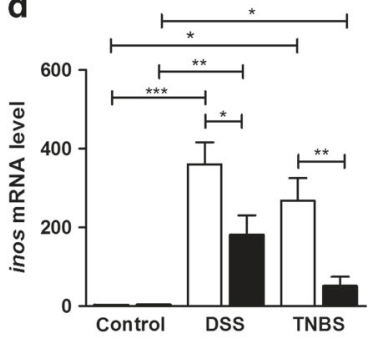

g

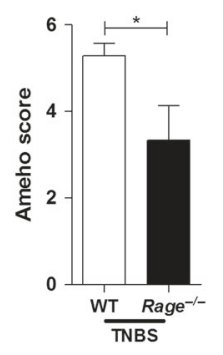

h $\quad$ WT

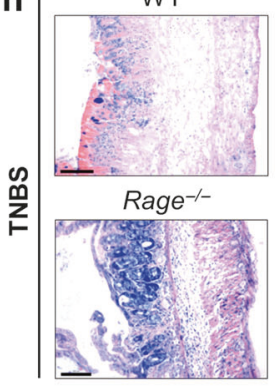

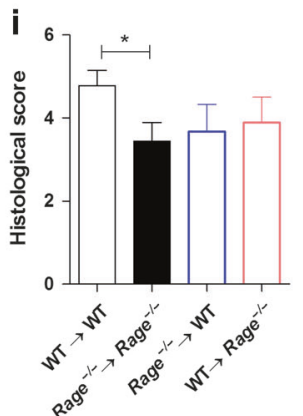

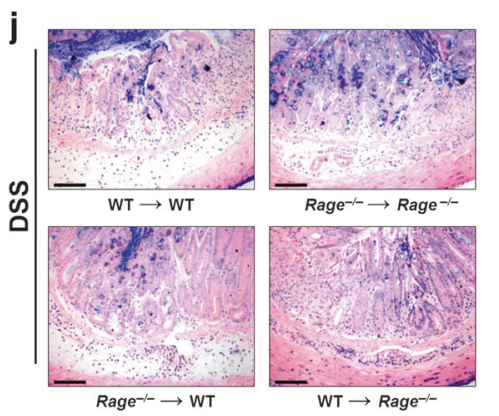

k

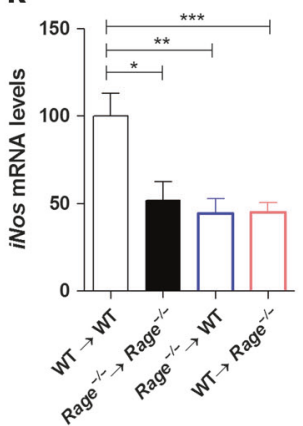

I

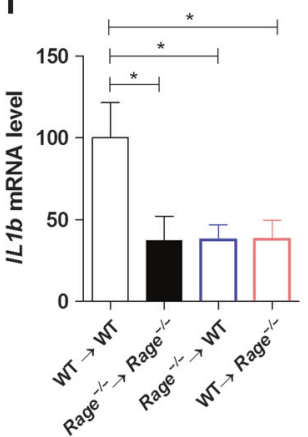

m

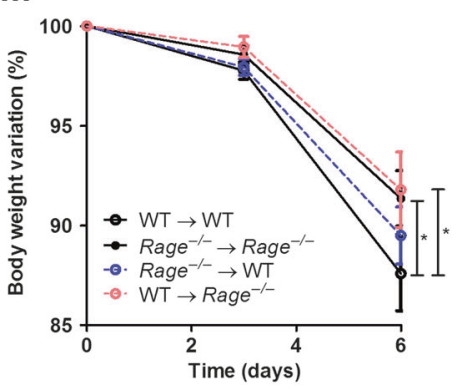

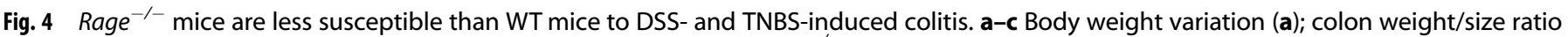
(b); and quantification of colonic MPO activity (c) of WT $(n=10)$ and Rage ${ }^{-1-}(n=12)$ mice administered with $2.5 \%$ DSS for 8 days. iNos (d) and $I L 1 \beta$ (e) mRNA quantification in the colon of control, DSS- and TNBS-treated WT and Rage ${ }^{-/-}$mice. $\mathbf{f}-\mathbf{h}$ Colonic macroscopic (f) and Ameho (g) scores; and representative pictures of colonic lesions (scale bar, $100 \mu \mathrm{m})(\mathbf{h})$ of WT $(n=5)$ or Rage ${ }^{-/-}(n=6)$ mice $48 \mathrm{~h}$ after TNBS treatment. WT and Rage ${ }^{-/-}$bone marrow (BM) chimeras were generated and DSS colitis was induced. i-m Histological score (i); representative pictures of colonic sections (scale bar, $100 \mu \mathrm{m})(\mathbf{j}) ;$ iNos $(\mathbf{k})$ and IL1 $\beta$ (I) mRNA quantification in the colon; and body weight variation ( $\mathbf{m})$ of WT $\rightarrow$ WT $(n=$ 9), Rage $^{-/-} \rightarrow$ Rage $^{-/-}(n=10)$, Rage $^{-/-} \rightarrow$ WT $(n=10)$ and WT $\rightarrow$ Rage $^{-/-}(n=9)$ mice. For both DSS and TNBS, data are from three independent experiments. ${ }^{*} p<0.05,{ }^{* *} p<0.01,{ }^{* * *} p<0.005$ determined by Mann-Whitney test

activity (Fig. 6f) and iNOS and IL1 $\beta$ mRNA levels (Fig. 6g, h) were decreased after FPS-ZM1 treatment in treated DSS mice compared to untreated DSS mice. Finally, mice treated with DSS and RAGE inhibitor did not exhibited differences, for all the studied parameters, with mice not treated with DSS, showing a full protection of colitis development by FPS-ZM1 treatment. Together, our results demonstrate that targeting RAGE strongly decreases small bowel inflammation and abolishes colitis development, arguing in favor of the use of RAGE as a new therapeutic target in IBD.

\section{RAGE-dependant endothelial activation mediates colitis development}

As RAGE is expressed in endothelial cells and is suggested to play a role in vascular dysfunction and as endothelial dysfunction is a key feature in IBD development, we tested the impact of RAGE deficiency in endothelial activation during intestinal inflammation. ${ }^{30-32}$ We previously showed that Rage $^{-1-}$ mice expressed less iNos compared to WT mice during DSS and TNBS-induced colitis (Fig. 4d). We observed that expression of the cellular adhesion molecule Icam-1 and the endothelial cells proliferating factor Igf-1 were decreased in Rage ${ }^{-/-}$mice compared to WT mice under DSS and TNBS colitis (Fig. 7a). We also showed a decreased expression of the neoangiogenic factor KC in Rage ${ }^{-/-}$mice compared to WT mice (Fig. 7b). Together, these results suggested that the reduction of endothelial activation by RAGE deficiency may partly account for the reduction of colitis development. We then tested the effect of the therapeutic inhibition of RAGE on endothelial dysfunction. We already showed that FPS-ZM1 administration decreased the DSS-induced expression iNos (Fig. 6g). We then evidenced that FPS-ZM1 diminished the DSS-induced expression of Icam-1, Igf-1, and KC (Fig. 7c). Blocking RAGE in vivo, reduced endothelial activation which may be a mechanism for the protection during colitis development.

\section{DISCUSSION}

Inflammatory Bowel Diseases (IBD) including Crohn's disease (CD) and ulcerative colitis (UC) are inflammatory conditions of the colon and small intestine. CD can affect the small intestine and 
a

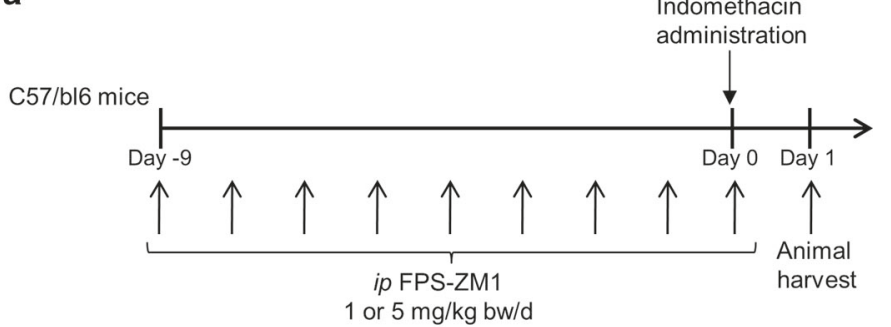

b

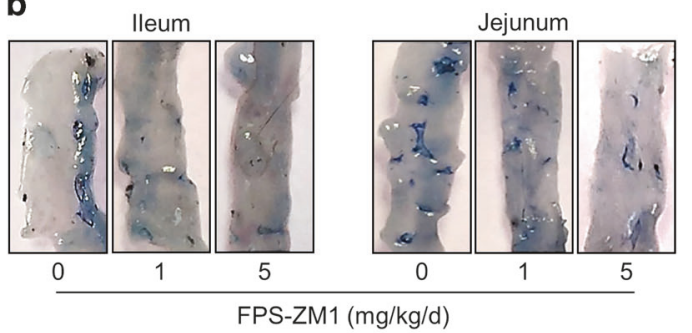

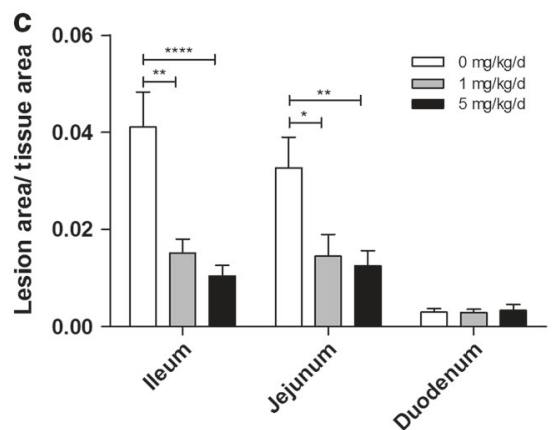

f

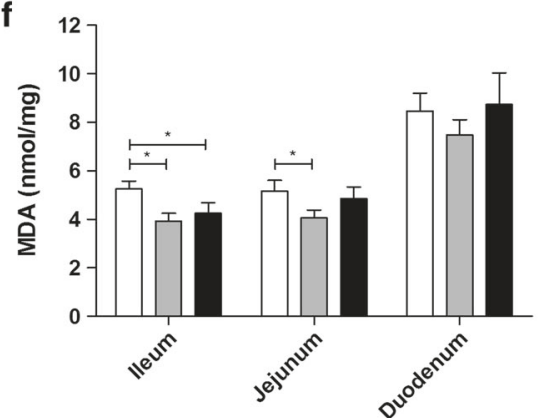

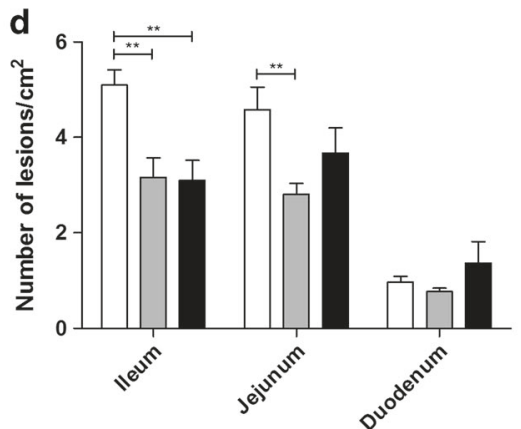

g

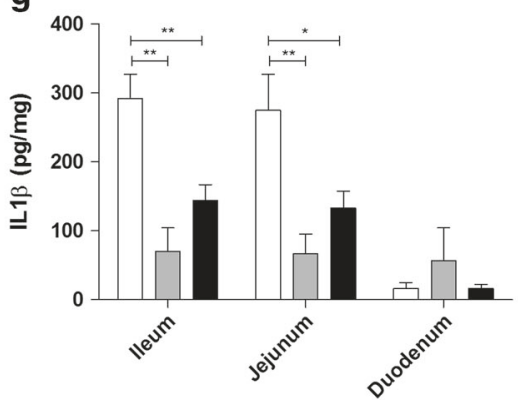

e

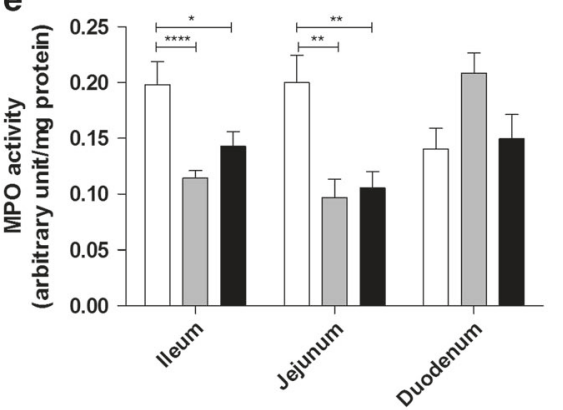

h

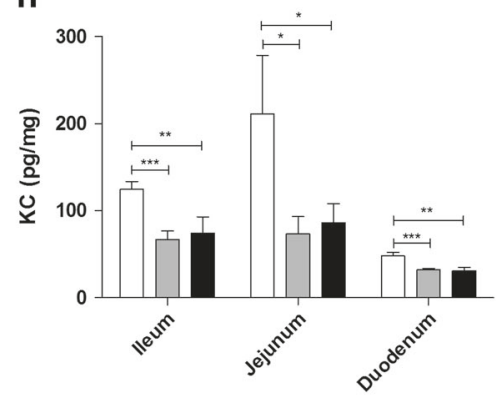

Fig. 5 The RAGE inhibitor FPS-ZM1 protects mice from indomethacin-induced enteritis. a Study design. $\mathbf{b}$ Representative pictures of ileal and jejunal lesions. c-h Area (c); and number (d) of lesions; quantification of MPO activity (e); and MDA (f); ELISA quantification of IL1 $\beta$ (g); and KC (h); in the ileum, jejunum and duodenum, of FPS-ZM1-treated and untreated mice with indomethacin-induced enteritis ( $n=10$ per group). Data are from two independent experiments. ${ }^{*} p<0.05,{ }^{* *} p<0.01,{ }^{* * *} p<0.005,{ }^{* * *} p<0.001$ determined by Mann-Whitney test

colon, and also but less frequently, the entire digestive tract from the mouth to the anus, whereas UC affects the colon and the rectum. IBD are believed to result from a complex interplay between genetic susceptibility, host-microbe interaction and environmental factors. ${ }^{33}$ The strategy in the treatment of IBD is to control the gastrointestinal inflammation, with the ultimate goal to achieve mucosal healing, signature for a good long-term prognosis, such as low risk of recurrence or surgery. Although the introduction of monoclonal antibodies targeting TNF- $a$ has revolutionized treatment for $I B D$, many patients do not respond or progressively lose response to these drugs. Advances of knowledge in pathophysiology of gut inflammatory processes will made possible the identification of new molecular targets for drugs. The global aim of our study was to assess the role of the RAGE signaling pathway in the pathogenesis of intestinal diseases and the opportunity of a RAGE-based therapeutic intervention in IBD.

Our study first showed that RAGE was sparsely expressed in the small intestine and in the colon under steady state conditions and was upregulated during inflammation. Our results in human extend previous investigations, which found an increase of RAGE expression during $C^{23,24}$ In the present study, samples from Ulcerative Colitis patients were added and $C D$ patients were stratified according to disease localization (i.e., colonic vs ileal). Moreover, a quantitative method based on ELISA quantification of RAGE protein was included showing a positive correlation between tissue levels of RAGE and the inflammatory status. In mice, RAGE can be detected in the ileum and the colon in control animals and is upregulated during inflammation with the exception of the duodenum in indomethacin-treated mice. These results are explained by the injury preferentially induced in the ileum and jejunum but not in the duodenum by indomethacin treatment.

In order to evaluate the potential role of the RAGE signaling pathway during intestinal inflammation development, we applied the well-described models of IBD, namely TNBS, DSS and indomethacin models, in WT and Rage ${ }^{-/-}$mice. ${ }^{34-37}$ We found that RAGE deficiency protected mice against enteritis as reflected by a diminished number and severity of necrotizing lesions induced by indomethacin and decreased inflammatory and oxidative markers in the small intestine. Rage $^{-1-}$ mice were also more resistant to colitis development as observed by improved clinical and macroscopic parameters together with a less proinflammatory and pro-oxidative environment. The results obtained in chimeric mice demonstrated that the simultaneous expression of RAGE in immune and non-immune cells is important for colitis development. The importance of RAGE expression in immune cells is consistent with the massive infiltrate observed during intestinal inflammation. Our results on the implication of RAGE in indomethacin-induced small intestine inflammation are not in agreement with previous report showing an absence of protection in Rage $^{-/-}$mice. ${ }^{27}$ This can be explained by the 
a
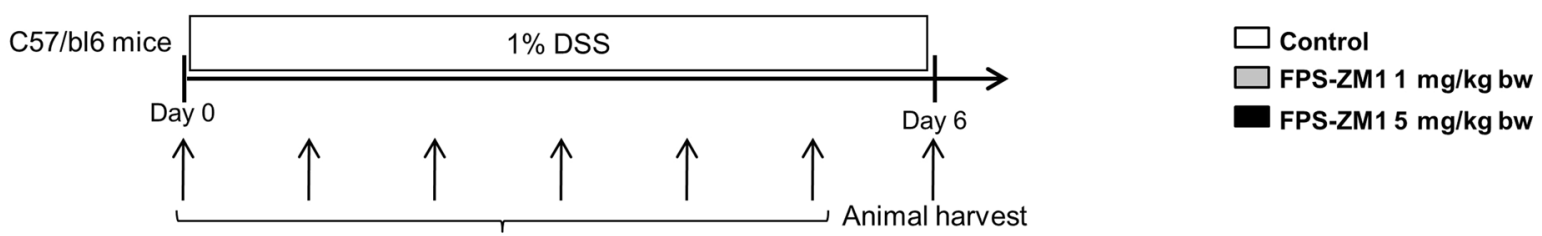

ip FPS-ZM1

1 or $5 \mathrm{mg} / \mathrm{kg} \mathrm{bw} / \mathrm{d}$

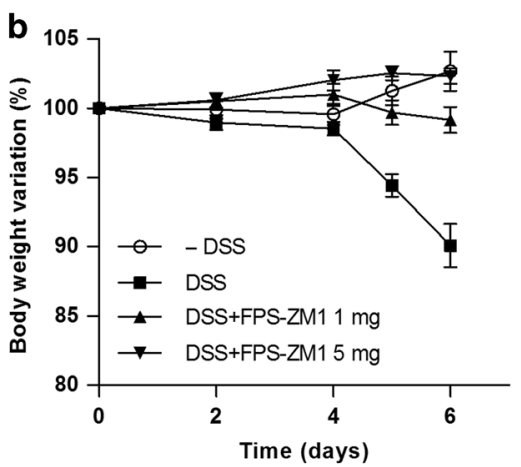

C

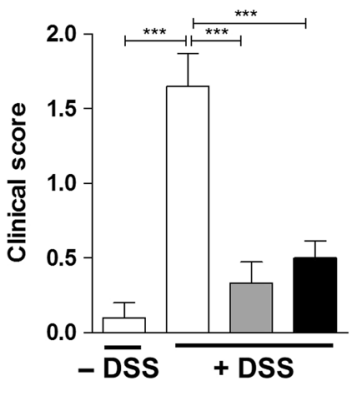

d

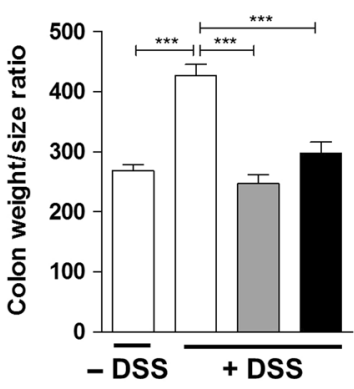

e

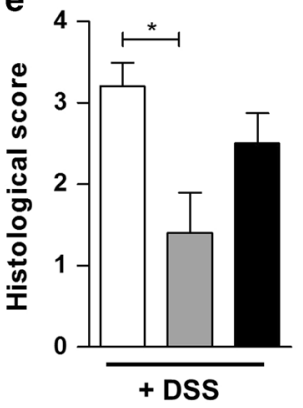

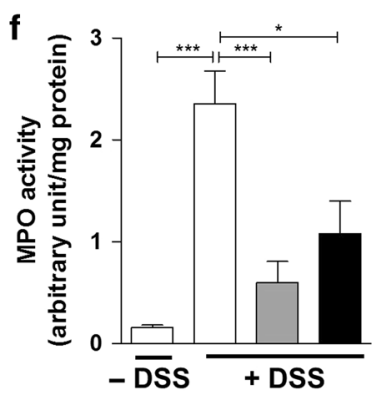
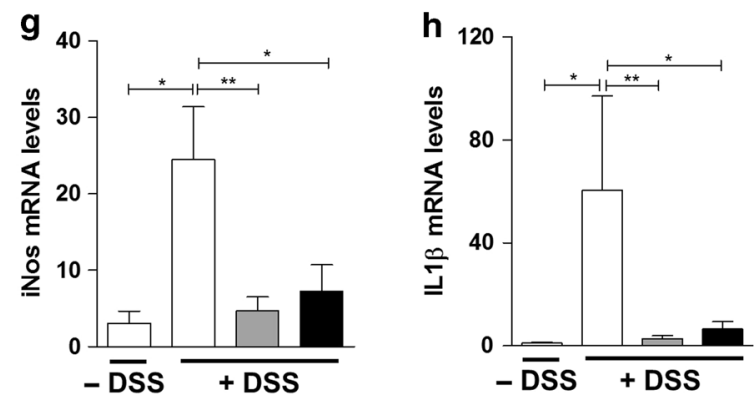

Fig. 6 The RAGE inhibitor FPS-ZM1 protects mice from DSS-induced colitis. a Study design. b-h Body weight variation (b); clinical score (c); colon weight/size ratio (d); Histological score (e); quantification of colonic MPO activity (f); and iNos (g) and II1 $\beta$ (h) mRNA quantification in the colon of control mice without DSS (-DSS, $n=5)$ and of FPS-ZM1-treated and untreated mice with DSS-induced colitis ( + DSS, $n=10)$. Data are from two independent experiments. ${ }^{*} p<0.05,{ }^{* *} p<0.01,{ }^{* * *} p<0.005$ determined by Mann-Whitney test. See Fig. S4 for body weight variation statistical analysis

difference in the RAGE knockdown constructs though both constructs result in absence of RAGE mRNA and protein expressions. $^{38,39}$ Moreover, the dose of indomethacin used was not the same (10 mg/kg vs $20 \mathrm{mg} / \mathrm{kg}$ in our study), and the small intestine was analyzed by segment in our study and globally in the study by Nadatani and coll. Finally, and maybe more importantly, indomethacin treatment did not induce small intestinal RAGE expression in the study by Nadatani and coll., whereas an increased RAGE mRNA and protein levels were observed in the ileum and jejunum in our study (Fig. 2a, b). Our results in the protection of Rage $^{-1-}$ mice in the DSS model are not in agreement with a previous study showing no difference in severity of colitis, which however used the same mouse colony. ${ }^{24}$ The major difference between the two studies lies on the experimental protocol used, with colonic inflammation studied during the acute phase of colitis induction in our model compared to the late phase during the recovery process in the study by Däbritz and coll.

The involvement of RAGE as a central activator of inflammation has already been demonstrated in other chronic inflammatory diseases. RAGE ligands are generated in vivo during inflammation and oxidative stress (such as during IBD) contributing to the persistence of the inflammatory process and the subsequent tissue damage. ${ }^{19,25,26,40}$ RAGE has been linked to the complications of diabetes such as accelerated atherosclerosis, nephropathy and retinopathy. ${ }^{17,18}$ The implication of RAGE has also been described in the pathogenesis of neurodegenerative diseases, Alzheimer's disease, Parkinson's disease or amyloid lateral sclerosis. ${ }^{18}$ Although less documented, the pathophysiology of cardiac disorders also seems to involve the RAGE signaling pathway. ${ }^{41}$ Inflammation and cancer sharing many signaling pathways, the role of RAGE in carcinogenesis and the link with cell transformation, apoptosis and metastasis has been established. ${ }^{16,42,43}$ Therefore, RAGE has become an attractive target for therapeutic purpose. ${ }^{7}$ In this study, we found that administration of FPS-ZM1, a specific inhibitor of RAGE, resulted in improvements of gut damage during enteritis and colitis development. Increased expression of pro-inflammatory cytokines and oxidative stress observed during these conditions were suppressed by FPS-ZM1 administration. This suggests that the protective effect of FPS-ZM1 is achieved by reducing the inflammatory and oxidative responses.

RAGE signaling has been linked to endothelial activation and dysfunction in a number of studies. ${ }^{30,31}$ Endothelial dysfunction plays an important role in IBD development. ${ }^{44}$ Adhesion molecules (ICAM-1, VCAM-1) are upregulated in IBD patients and in experimental models of colitis and play a key role in leukocytes recruitment and production of mediators of inflammation and angiogenesis at the inflammatory site. ${ }^{45}$ INOS derived NO is an important factor involved in pathological angiogenesis and contributes to inflammatory cells recruitment by increasing the expression of endothelial adhesion molecules. We demonstrated 
a
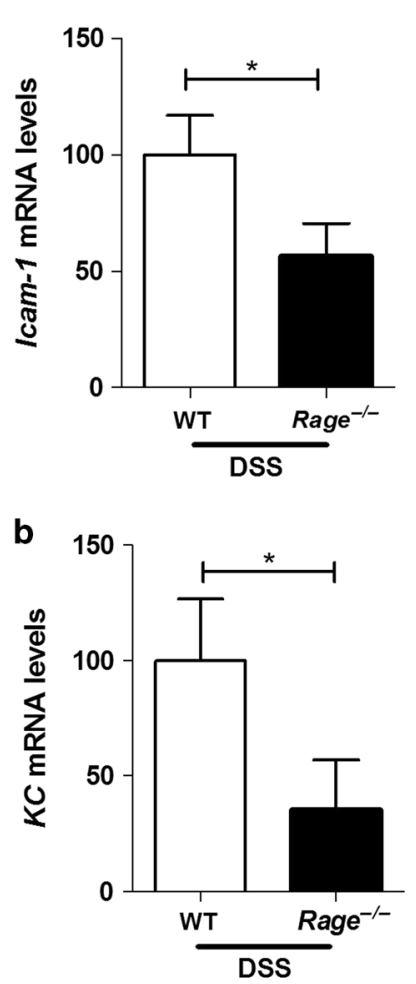

C

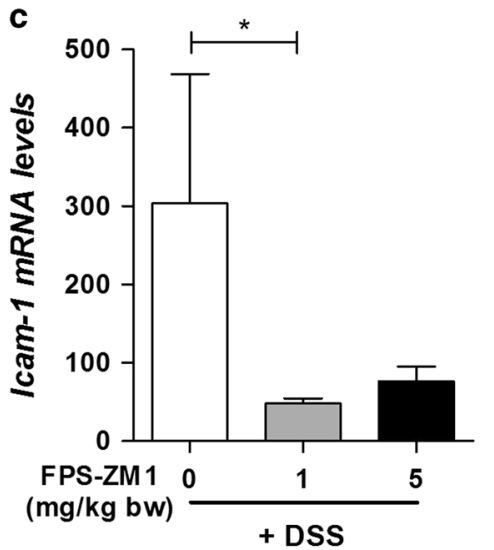

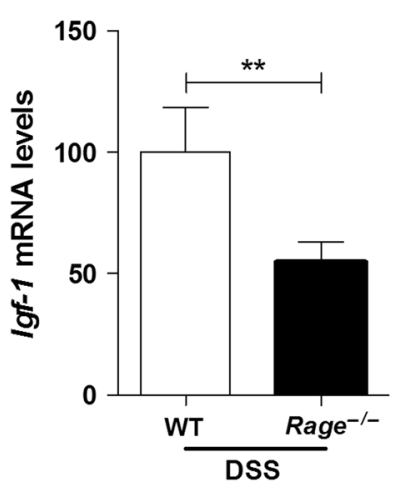
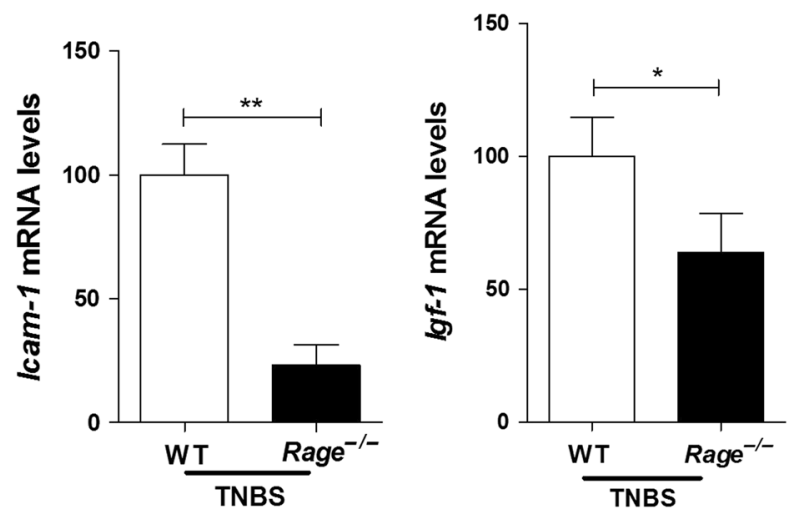
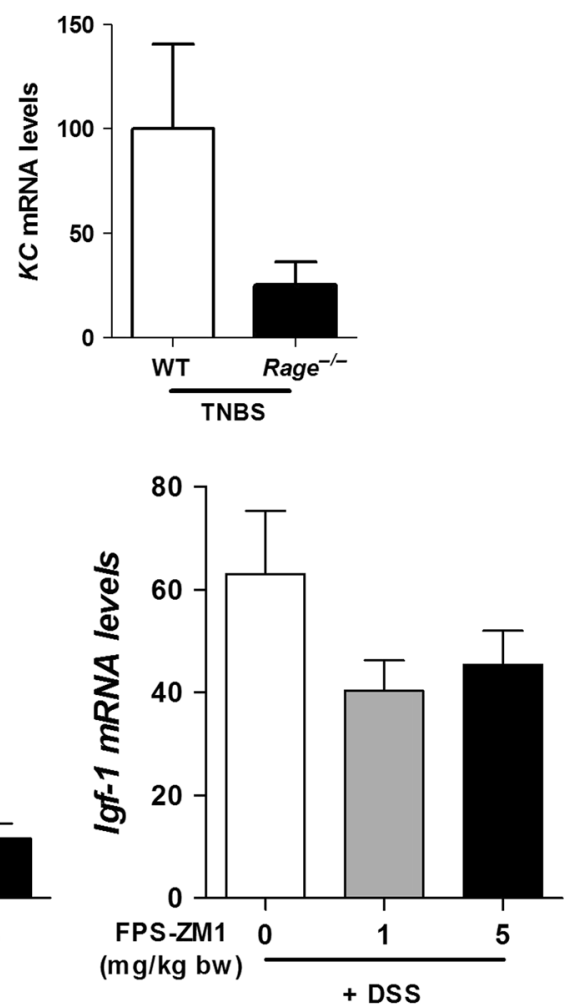

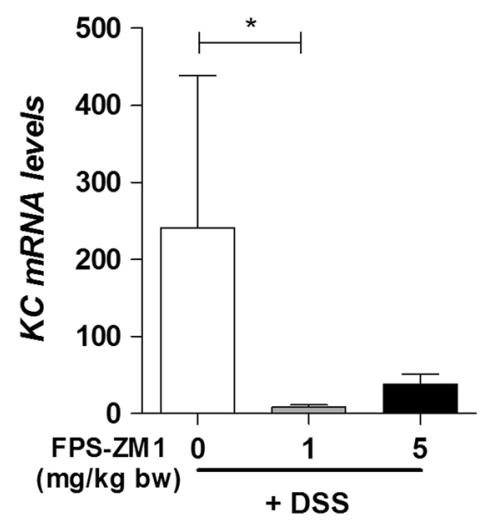

Fig. 7 RAGE-dependant endothelial activation mediates colitis development. Icam-1 and Igf-1 (a) and KC (b) mRNA quantification in the colon of WT $(n=10)$ and Rage $e^{-1-}(n=12)$ mice administered with $2.5 \%$ DSS for 8 days, and of WT $(n=5)$ or Rage $e^{-/-}(n=6)$ mice $48 \mathrm{~h}$ after TNBS treatment. Data are from 3 independent experiments. Icam-1, Igf-1, and KC (c) mRNA quantification in the colon of FPS-ZM1-treated and untreated mice with DSS-induced colitis $(n=10)$. Data are from 2 independent experiments. ${ }^{*} p<0.05,{ }^{* *} p<0.01$ determined by Mann-Whitney test

here that RAGE deficiency or blockage of RAGE by the FPS-ZM1 inhibitor decreased adhesion molecule, angiogenic factor, oxidative stress, and inflammatory mediators expression, all involved in endothelial activation and dysfunction during colitis. One mechanism by which RAGE activation might affect the intestinal inflammation is by amplifying expression of inflammatory mediators and adhesive receptors promoting leukocyte recruitment. Our results are in agreement with recent findings suggesting a role for RAGE in the activation of the lung endothelium contributing to the development of asthma/allergic airway inflammation. ${ }^{46}$

The FPS-ZM1, whose full chemical name is $N$-Benzyl- $N$ cyclohexyl-4-chlorobenzamide, binds specifically to the variable Immunoglobulin-like domain (V domain) of RAGE. ${ }^{29}$ FPS-ZM1 has been experimentally demonstrated to block RAGE binding to $A \beta$,
S100B, and HMGB1, but might block binding to AGEs or S100P proteins, ligands that also interact with the $\mathrm{V}$ domain of RAGE. ${ }^{7}$ FPS-ZM1-RAGE targeting has been shown to be efficient in several other preclinical models with few toxic long-term side effects. ${ }^{47-50}$ Other synthetic small-molecule inhibitors of RAGE have been developed. ${ }^{7}$ Among them, Azelarigon (also called PF-04494700 or TTP488) inhibits RAGE binding to $A \beta, S 100 B, H M G B 1$, and CML and showed improved performance in Alzheimer's disease mice models. ${ }^{51,52}$ Azelarigon successfully achieved a Phase II clinical trial offering a slowed cognitive decline for mild Alzheimer's disease patients, and a phase III trial is ongoing. ${ }^{53}$ Strategies using anti-RAGE antibodies or antagonistic peptides are also developed. ${ }^{54,55}$ Our results represent a proof of principle study showing that antagonizing RAGE might be an efficient therapeutic strategy in IBD. 


\section{METHODS}

Human samples

Human gut samples were collected upon surgical resection. The study was approved by the local ethics committee (Comité de Protection des Personnes Nord Ouest IV, CHRU Lille, France, No. DC-2008-642). All the subjects gave their informed consent. The characteristics of patient cohort are detailed in Supplementary Table 1.

\section{Mice}

All animal procedures were conducted in accordance with the institutional guidelines approved by the institutional Animal Care and Ethical Use Committee of the University of Lille (committee no. 75; authorization no. CEEA2016100410284439). Rage Re mice $^{-/-}$ were generated as previously described, ${ }^{39}$ backcrossed to $\mathrm{C} 57 \mathrm{BL} / 6$ for more than 12 generations and the colony (Rage ${ }^{-1-}$ and WT) was bred in-house at the animal facility of Lille University Hospital. C57BL/6 mice were obtained from Janvier Labs. Male and female mice from 7 to 12 weeks old were used in the study. Age and sex matched mice were used in all experiments. Mice were randomly assigned to experimental groups.

Immunofluorescence staining of human and mice tissues Human and mice samples were fixed for one night in $4 \%$ formaldehyde, dehydrated successively in ethanol and xylene, and then embedded in paraffin. $4 \mu \mathrm{m}$ sections were cut on a Microtome Leica RM2245 and laid on Superfrost Plus slides. For Immunofluorescence staining, epitope retrieval was performed in $0.01 \mathrm{M}$ Tris-sodium citrate (Sigma-Aldrich), pH 6, in a $600 \mathrm{~W}$ microwave for $20 \mathrm{~min}$. After cooling and rinsing in PBS, $0.1 \%$ Tween 20, sections were blocked in PBS, 0.1\% Tween20, 5\% BSA. Sections were stained with a goat anti-RAGE antibody (Abcam) and, for human sections, a rabbit anti-keratin 20 antibody (Cell Signaling Technology). Detection was performed with an Alexa fluor 488-conjugated anti-goat antibody and an Alexa fluor 647conjugated anti-rabbit antibody (Thermo Fisher Scientific). Nuclear staining with Hoechst was performed before adding a fluorescent mounting medium. For haematoxylin eosin staining, briefly, sections were stained in Harris haematoxylin for $8 \mathrm{~min}$, rinse in water then in $95 \%$ alcohol and counterstained in eosin $Y$ solution for $1 \mathrm{~min}$. For human samples, microscopy was performed using a slide scanner (Axioscan Z1), and data were processed with ZEN 2010 software. For mice samples, microscopy was performed using a microscope Leica DM5500 B and data were processed with the Leica LAS V3.8 software.

\section{Protein extraction for ELISA quantification}

Human and mice tissues were immediately frozen after sampling and proteins were extracted using TRlzol ${ }^{\circledR}$ Reagent according to the manufacturer (Thermo Fisher Scientific). Briefly, tissues were homogenized in TRIzol $^{\circledR}$ Reagent with a Precellys ${ }^{\circledR} 24$ homogenizer (Bertin Technologies) and chloroform was added separating DNA and proteins in an organic layer. Proteins were precipitated by isopropanol, washed with $0.3 \mathrm{M}$ guanidine hydrochloride in $95 \%$ ethanol and $100 \%$ ethanol. Proteins were then resuspended in 1\% SDS, $8 \mathrm{M}$ urea (Sigma-Aldrich). The total quantity of proteins was determined using the $D C^{T M}$ protein assay kit (Bio-Rad) and human ELISA for RAGE (R\&D Systems), IL8 and IL1 $\beta$ (Thermo Fisher Scientific) and mice ELISA for RAGE, KC (R\&D Systems) and IL1 $\beta$ (Thermo Fisher Scientific) were performed as described by the manufacturer.

Induction and macroscopic evaluation of small intestinal inflammation induced by indomethacin

To induce small intestinal injury, $20 \mathrm{mg} / \mathrm{kg}$ bw indomethacin (Sigma-Aldrich) dissolved in $0.5 \%$ carboxymethyl cellulose (CMC) was orally administered to mice. ${ }^{35-37}$ At the end of the experiment ( $24 \mathrm{~h}$ after indomethacin injection), mice were intravenously administered with $1 \%$ Evans blue (Sigma-Aldrich) in $0.9 \% \mathrm{NaCl}$ and sacrificed $30 \mathrm{~min}$ later. Evans blue is used to visualize macroscopic lesions. At necropsy, ileum, jejunum and duodenum were separately harvested, longitudinally opened and photographed. Tissues were then immediately frozen for subsequent analysis. The number and area of lesions and tissues were quantified using Image J software.

Induction and macroscopic evaluation of colonic injury induced by dextran sulfate sodium (DSS)

Colitis was induced by adding $2.5 \%$ (w/v) DSS (TdB Consultancy) in the drinking water of mice for 8 days. For experiments using FPSZM1, colitis was induced with 1\% DSS for 6 days. Fresh DSS solutions were prepared every two days. Mice body weight was recorded during the experiment. Clinical score calculation is based on the scoring of the stool consistency and occult blood. The presence of blood in the stool was assessed with ColoScreen Occult blood test (ELITech Group). At necropsy, colon was carefully dissected and its weight and size were measured. Colon was then either immediately frozen or fixed in $4 \%$ formaldehyde (VWR Chemicals).

Induction and macroscopic evaluation of colonic injury induced by 2,4,6-trinitrobenzene sulfonic acid (TNBS)

TNBS colitis was induced in anesthetised C57BL/6 mice by intrarectal administration of TNBS $(150 \mathrm{mg} / \mathrm{kg})$ (Sigma-Aldrich) diluted in a $1: 1(\mathrm{v} / \mathrm{v})$ mix of $0.9 \% \mathrm{NaCl}$ and $100 \%$ ethanol as described previously. ${ }^{34}$ Control animals received $\mathrm{NaCl} /$ ethanol mix using the same technique. Mice were euthanized 4 days after TNBS/ethanol administration. The colon of each mouse was dissected and cut longitudinally to reveal the colonic mucosa. Intensity of colonic lesions was evaluated macroscopically according to the Wallace score. The Wallace score rates macroscopic lesions on a scale from 0 to 10 based on features reflecting inflammation, such as hyperemia, thickening of the bowel, and extent of ulceration. A colon specimen located within the ulceration was frozen for subsequent analysis of mRNA expression.

Generation of bone marrow chimeras WT and Rage $^{-/-}$recipient mice were exposed to two doses of 5 $\mathrm{Gy}, 4 \mathrm{~h}$ apart. Twenty-four hours later, irradiated recipient mice were reconstituted by intravenous injection of $10 \times 10^{6} \mathrm{BM}$ cells, obtained from femurs and tibias of donor mice, resuspended in $0.1 \mathrm{ml}$ of $0.9 \% \mathrm{NaCl}$. Chimeric mice were provided with $0.2 \mathrm{mg} / \mathrm{ml}$ ciprofloxacin (Sigma-Aldrich) for 3 weeks after BM transplantation and housed for 6 weeks before DSS treatment. Efficient reconstitution by donor bone marrow cells was confirmed by PCR for the Rage gene in splenocytes from BM chimeric mice.

FPS-ZM1 preparation and administration

FPS-ZM1 (Merck Millipore) was diluted in $150 \mathrm{mM}$ cyclodextrin (Sigma-Aldrich) prepared in sterile water, and intraperitoneally administered to mice at a final concentration of 1 or $5 \mathrm{mg} / \mathrm{kg} \mathrm{bw} /$ day. Control mice were injected with cyclodextrin.

May-Grünwald and Giemsa staining of intestinal tissues from mice and histological evaluation

Colon, ileum or jejunum samples were fixed in $4 \%$ formaldehyde for one night, and then embedded in paraffin. Tissue sections were stained with May-Grünwald and Giemsa (MGG) (Carlo Erba reagents) and evaluated blindly by two investigators. In TNBSinduced colitis, histological lesions were quantified in the colon using the modification by Ameho of the histopathological grading system of Macpherson and Pfeiffer (ranging from 0 to 6). Briefly, histological findings identical to normal mice were scored as 0 , mild mucosal and/or submucosal inflammatory infiltrate and edema, punctuate mucosal erosions, and muscularis mucosae 
intact were scored as 1 , the same histological findings involving $50 \%$ of the specimen were scored as 2, prominent inflammatory infiltrate and edema, deeper areas of ulceration extending through the muscularis mucosae into the submucosa, and rare inflammatory cells invading the muscularis propriae but without muscle necrosis were scored as 3 , the same histological findings involving $50 \%$ of the specimen were scored as 4 , extensive ulceration with coagulative necrosis with deep extension of the necrosis into the muscularis propria were scored as 5 , the same histological findings involving $50 \%$ of the specimen were scored as 6.

In DSS-induced colitis experiments, histological lesions were assessed using a score quantifying the intensity of the inflammatory cell infiltrate (score $0-3$ ) and the tissue damage (score 0-3). Briefly, the presence of occasional inflammatory cells in the lamina propria was scored as 0 , increased numbers of inflammatory cells in the lamina propria as 1, confluence of inflammatory cells extending into the submucosa as 2 , and transmural extension of the infiltrate as 3 . For tissue damage, scores were: 0 , no mucosal damage; 1 , lymphoepithelial lesions; 2 , surface mucosal erosion or focal ulceration; 3, extensive mucosal damage and extension into deeper structures of the bowel wall. The combined histological score ranged from 0 (no changes) to 6 (extensive infiltration and tissue damage).

Determination of myeloperoxidase (MPO) activity

MPO is a protein found in granules of neutrophils, its quantification is used as a marker of inflammation. Small intestine and colon specimens were homogenized with a Precellys ${ }^{\circledR} 24$ homogenizer in a phosphate buffer ( $\mathrm{pH} 6.0)$ containing $0.5 \%$ hexadecyltrimethyl ammonium (Sigma-Aldrich), and subjected to two sonication and freeze-thaw cycles. The suspensions were centrifuged at $14,000 \times$ $g$ for $15 \mathrm{~min}$ at $4{ }^{\circ} \mathrm{C}$ and the supernatants were reacted with $1 \mathrm{mg} /$ $\mathrm{ml}$ o-dianisidine hydrochloride and $0.0005 \%$ hydrogen peroxide. Optical density of each sample was read at $450 \mathrm{~nm}$ with a Versamax microplate reader (MDS analytical technologies). One unit of MPO activity was defined as the amount that degraded 1 $\mu \mathrm{mol}$ peroxidase per minute at $25^{\circ} \mathrm{C}$. The results were expressed as absorbance per total quantity of proteins determined using the $\mathrm{DC}^{\mathrm{TM}}$ protein assay kit.

\section{Assessment of lipid peroxidation}

Quantification of malondialdehyde (MDA) is used as a marker of lipid peroxidation. Tissues were homogenized with a Precellys ${ }^{\circledR} 24$ homogenizer (Bertin Technologies) in cold Phosphate-Buffered Saline and centrifuged at $10,000 \times g$ for $15 \mathrm{~min}$ at $4^{\circ} \mathrm{C}$. Supernatants were incubated with acetic acid, 2-Thiobarbituric acid and SDS at $95^{\circ} \mathrm{C}$ for $1 \mathrm{~h}$. Supernatants were transferred to a 96-well plate and the fluorescence was measured at $\lambda_{\mathrm{ex}}=532$ and $\lambda_{\mathrm{em}}=$ $553 \mathrm{~nm}$ with a microplate reader fluostar omega (BMG Labtech). 1,1,3,3-Tetramethoxypropane was used as a standard. Protein concentration in samples was determined using a $\mathrm{DC}^{\mathrm{TM}}$ protein assay (Bio-Rad).

Real-time quantitative PCR

Total RNA was extracted from small intestine and colonic samples with the NucleoSpin RNAll commercial kit as described by the manufacturer (Macherey-Nagel). cDNA was synthesized with the High Capacity cDNA Reverse Transcription kit and qPCR was performed with SyBrGreen fluorescence detection in a StepOne system (Thermo Fisher Scientific). The primer sequences were designed using Primer Express 3 and are available upon request. Melting curve analyses were performed for each sample and gene to confirm the specificity of the amplification. The relative expression of each target gene was normalized to the relative expression of the Polr2a gene. The quantification of the target gene expression was based on the comparative cycle threshold
$(\mathrm{Ct})$ value. The fold changes in the target genes were analyzed by the $2^{-\Delta \Delta C t}$ method.

Statistical analysis

Data are presented as the mean \pm SEM. Comparison between different treatment groups was performed using the Mann-Whitney test. Analyzes were performed using the GraphPad Prism5 Software. Statistical significance was defined as $p<0.05$. For all experiments, ${ }^{*} p<0.05,{ }^{* *} p<0.01$, ${ }^{* *} p<0.005,{ }^{* * * *} p<0.001$. Number of samples and animals was indicated as " $n$ " and described in figure legends. Number of independent experiments are indicated in figure legends.

\section{ACKNOWLEDGEMENTS}

We thank Charbel Charter, Amélie Chau, and Lucil Schneider for their contribution in obtaining human samples. We thank Meryem Tardivel of the Bioimaging center of Lille Facility for access to systems and technical advice. We thank the animal facility of Lille University Hospital for the animal care and Cyrille Degraeve for breeding of RAGE colony. We thank Dr Thierry Idziorek of the University of Lille irradiation facility for access to instrument and technical advices. This work was supported by grants from the French association François Aupetit and the European Foundation DIGESTSCIENCE.

\section{AUTHOR CONTRIBUTIONS}

Study concept and design: M.B.M. and C.V.; acquisition, analysis, and interpretation of data: M.B.M., M.D., C.W., and C.V.; resources: P.Z., A.L., and A.M.S.; drafting of the manuscript: C.V.; revision of the manuscript: M.B.M., P.D., E.B., and C.G.; funding acquisition: P.D. and C.V.; study supervision: C.V.

\section{ADDITIONAL INFORMATION}

The online version of this article (https://doi.org/10.1038/s41385-018-0119-z) contains supplementary material, which is available to authorized users.

Competing interests: The authors declare no competing interests.

\section{REFERENCES}

1. Kaplan, G. G. \& Ng, S. C. Understanding and preventing the global increase of inflammatory bowel disease. Gastroenterology 152, 313-321.e2 (2017).

2. Souza, H. S. Pde \& Fiocchi, C. Immunopathogenesis of IBD: current state of the art. Nat. Rev. Gastroenterol. Hepatol. 13, 13-27 (2016).

3. Bamias, G., Pizarro, T. T. \& Cominelli, F. Pathway-based approaches to the treatment of inflammatory bowel disease. Transl. Res. J. Lab. Clin. Med. 167, 104-115 (2016).

4. Targownik, L. E. et al. Temporal trends in initiation of therapy with tumor necrosis factor antagonists for patients with inflammatory bowel disease: a populationbased analysis. Clin. Gastroenterol. Hepatol. Off. Clin. Pract. J. Am. Gastroenterol. Assoc. 15, 1061-1070.e1 (2017).

5. Colombel, J.-F., Narula, N. \& Peyrin-Biroulet, L. Management strategies to improve outcomes of patients with inflammatory bowel diseases. Gastroenterology 152, 351-361.e5 (2017).

6. Pouillon, L., Bossuyt, P. \& Peyrin-Biroulet, L. Considerations, challenges and future of anti-TNF therapy in treating inflammatory bowel disease. Expert. Opin. Biol. Ther. 16, 1277-1290 (2016).

7. Bongarzone, S., Savickas, V., Luzi, F. \& Gee, T. Targeting the receptor for advanced glycation endproducts (RAGE). J. Med. Chem. https://doi.org/10.1021/acs. jmedchem.7b00058 (2017).

8. Bopp, C. et al. Bench-to-bedside review: the inflammation-perpetuating patternrecognition receptor RAGE as a therapeutic target in sepsis. Crit. Care Lond. Engl. 12, 201 (2008).

9. Boulanger, E. et al. AGEs bind to mesothelial cells via RAGE and stimulate VCAM-1 expression. Kidney Int. 61, 148-156 (2002).

10. Butscheid, M., Hauptvogel, P., Fritz, P., Klotz, U. \& Alscher, D. M. Hepatic expression of galectin-3 and receptor for advanced glycation end products in patients with liver disease. J. Clin. Pathol. 60, 415-418 (2007).

11. Schmidt, A. M., Yan, S. D., Yan, S. F. \& Stern, D. M. The biology of the receptor for advanced glycation end products and its ligands. Biochim. Biophys. Acta 1498, 99-111 (2000). 
12. Schmidt, A. M. et al. Isolation and characterization of two binding proteins for advanced glycosylation end products from bovine lung which are present on the endothelial cell surface. J. Biol. Chem. 267, 14987-14997 (1992).

13. Du Yan, S. et al. Amyloid-beta peptide-receptor for advanced glycation endproduct interaction elicits neuronal expression of macrophage-colony stimulating factor: a proinflammatory pathway in Alzheimer disease. Proc. Natl Acad. Sci. USA 94, 5296-5301 (1997).

14. Hori, O. et al. The receptor for advanced glycation end products (RAGE) is a cellular binding site for amphoterin. Mediation of neurite outgrowth and coexpression of rage and amphoterin in the developing nervous system. J. Biol. Chem. 270, 25752-25761 (1995)

15. Hofmann, M. A. et al. RAGE mediates a novel proinflammatory axis: a central cell surface receptor for S100/calgranulin polypeptides. Cell 97, 889-901 (1999).

16. Ahmad, S. et al. AGEs, RAGEs and s-RAGE; friend or foe for cancer. Semin. Cancer Biol. https://doi.org/10.1016/j.semcancer.2017.07.001 (2017).

17. Ramasamy, R., Shekhtman, A. \& Schmidt, A. M. The multiple faces of RAGE opportunities for therapeutic intervention in aging and chronic disease. Expert. Opin. Ther. Targets 20, 431-446 (2016).

18. Ray, R., Juranek, J. K. \& Rai, V. RAGE axis in neuroinflammation, neurodegeneration and its emerging role in the pathogenesis of amyotrophic lateral sclerosis. Neurosci. Biobehav. Rev. 62, 48-55 (2016).

19. Sparvero, L. J. et al. RAGE (receptor for advanced glycation endproducts), RAGE ligands, and their role in cancer and inflammation. J. Transl. Med. 7, 17 (2009).

20. Xie, J., Méndez, J. D., Méndez-Valenzuela, V. \& Aguilar-Hernández, M. M. Cellular signalling of the receptor for advanced glycation end products (RAGE). Cell. Signal. 25, 2185-2197 (2013).

21. Bierhaus, A. et al. Understanding RAGE, the receptor for advanced glycation end products. J. Mol. Med. Berl. Ger. 83, 876-886 (2005).

22. Hofmann, M. A. et al. RAGE and arthritis: the G82S polymorphism amplifies the inflammatory response. Genes Immun. 3, 123-135 (2002).

23. Ciccocioppo, R. et al. Role of the advanced glycation end products receptor in Crohn's disease inflammation. World J. Gastroenterol. 19, 8269-8281 (2013).

24. Däbritz, J. et al. The functional -374T/A polymorphism of the receptor for advanced glycation end products may modulate Crohn's disease. Am. J. Physiol. Gastrointest. Liver Physiol. 300, G823-G832 (2011).

25. Foell, D. et al. Neutrophil derived human S100A12 (EN-RAGE) is strongly expressed during chronic active inflammatory bowel disease. Gut 52, 847-853 (2003).

26. Vitali, R. et al. Fecal HMGB1 is a novel marker of intestinal mucosal inflammation in pediatric inflammatory bowel disease. Am. J. Gastroenterol. 106, 2029-2040 (2011).

27. Nadatani, Y. et al. High mobility group box 1 promotes small intestinal damage induced by nonsteroidal anti-inflammatory drugs through Toll-like receptor 4 . Am. J. Pathol. 181, 98-110 (2012).

28. Davé, S. H. et al. Ethyl pyruvate decreases HMGB1 release and ameliorates murine colitis. J. Leukoc. Biol. 86, 633-643 (2009).

29. Deane, R. et al. A multimodal RAGE-specific inhibitor reduces amyloid $\beta$-mediated brain disorder in a mouse model of Alzheimer disease. J. Clin. Invest. 122, 1377-1392 (2012).

30. Skrha, J. et al. Relationship of soluble RAGE and RAGE ligands HMGB1 and ENRAGE to endothelial dysfunction in type 1 and type 2 diabetes mellitus. Exp. Clin. Endocrinol. Diabetes Off. J. Ger. Soc. Endocrinol. Ger. Diabetes Assoc. 120, 277-281 (2012).

31. Basta, G. et al. Advanced glycation end products activate endothelium through signal-transduction receptor RAGE: a mechanism for amplification of inflammatory responses. Circulation 105, 816-822 (2002).

32. Cibor, D. et al. Endothelial dysfunction in inflammatory bowel diseases: pathogenesis, assessment and implications. World J. Gastroenterol. 22, 1067-1077 (2016).
33. Khor, B., Gardet, A. \& Xavier, R. J. Genetics and pathogenesis of inflammatory bowel disease. Nature 474, 307-317 (2011).

34. Wirtz, S. et al. Chemically induced mouse models of acute and chronic intestinal inflammation. Nat. Protoc. 12, 1295-1309 (2017).

35. Xiao, X. et al. Gut microbiota mediates protection against enteropathy induced by indomethacin. Sci. Rep. 7, 40317 (2017).

36. Neurath, M. F. Animal models of inflammatory bowel diseases: illuminating the pathogenesis of colitis, ileitis and cancer. Dig. Dis. Basel Switz. 30(Suppl 1), 91-94 (2012).

37. Elson, C. O., Sartor, R. B., Tennyson, G. S. \& Riddell, R. H. Experimental models of inflammatory bowel disease. Gastroenterology 109, 1344-1367 (1995).

38. Myint, K.-M. et al. RAGE control of diabetic nephropathy in a mouse model: effects of RAGE gene disruption and administration of low-molecular weight heparin. Diabetes 55, 2510-2522 (2006).

39. Liliensiek, B. et al. Receptor for advanced glycation end products (RAGE) regulates sepsis but not the adaptive immune response. https://doi.org/10.1172/JCl18704 (2004).

40. Leach, S. T. et al. Serum and mucosal S100 proteins, calprotectin (S100A8/ S100A9) and S100A12, are elevated at diagnosis in children with inflammatory bowel disease. Scand. J. Gastroenterol. 42, 1321-1331 (2007).

41. Bangert, A. et al. Critical role of RAGE and HMGB1 in inflammatory heart disease. Proc. Natl Acad. Sci. USA 113, E155-E164 (2016).

42. Heijmans, J. et al. Rage signalling promotes intestinal tumourigenesis. Oncogene 32, 1202-1206 (2013)

43. Kang, R. et al. The receptor for advanced glycation end products (RAGE) sustains autophagy and limits apoptosis, promoting pancreatic tumor cell survival. Cell Death Differ. 17, 666-676 (2010).

44. Danese, S. Role of the vascular and lymphatic endothelium in the pathogenesis of inflammatory bowel disease: 'brothers in arms'. Gut 60, 998-1008 (2011).

45. Chidlow, J. H., Shukla, D., Grisham, M. B. \& Kevil, C. G. Pathogenic angiogenesis in IBD and experimental colitis: new ideas and therapeutic avenues. Am. J. Physiol. -Gastrointest. Liver Physiol. 293, G5-G18 (2007).

46. Perkins, T. N., Oczypok, E. A., Milutinovic, P. S., Dutz, R. E. \& Oury, T. D. RAGEdependent VCAM-1 expression in the lung endothelium mediates IL-33 induced allergic airway inflammation. Allergy (2018).

47. Gu, Q. et al. Contribution of receptor for advanced glycation end products to vasculature-protecting effects of exercise training in aged rats. Eur. J. Pharmacol. 741, 186-194 (2014)

48. Hong, Y. et al. Effects of RAGE-specific inhibitor FPS-ZM1 on amyloid- $\beta$ metabolism and AGEs-induced inflammation and oxidative stress in rat hippocampus. Neurochem. Res. 41, 1192-1199 (2016).

49. Kwak, T. et al. Targeting of RAGE-ligand signaling impairs breast cancer cell invasion and metastasis. Oncogene 36, 1559-1572 (2017).

50. Lee, H. et al. Blockade of RAGE ameliorates elastase-induced emphysema development and progression via RAGE-DAMP signaling. FASEB J. Off. Publ. Fed. Am. Soc. Exp. Biol. 31, 2076-2089 (2017).

51. Galasko, D. et al. Clinical trial of an inhibitor of RAGE-A $\beta$ interactions in Alzheimer disease. Neurology 82, 1536-1542 (2014).

52. Sabbagh, M. N. et al. PF-04494700, an oral inhibitor of receptor for advanced glycation end products (RAGE), in Alzheimer disease. Alzheimer Dis. Assoc. Disord. 25, 206-212 (2011).

53. Burstein, A. H. et al. Effect of TTP488 in patients with mild to moderate Alzheimer's disease. Bmc. Neurol. 14, 12 (2014).

54. Arumugam, T., Ramachandran, V., Gomez, S. B., Schmidt, A. M. \& Logsdon, C. D. S100P-derived RAGE antagonistic peptide reduces tumor growth and metastasis. Clin. Cancer Res. Off. J. Am. Assoc. Cancer Res. 18, 4356-4364 (2012).

55. Matsumoto, H. et al. Therapeutic effectiveness of anti-RAGE antibody administration in a rat model of crush injury. Sci. Rep. 7, 12255 (2017). 